\title{
How Hospitable Are Space Weather Affected Habitable Zones? The Role of Ion Escape
}

\author{
Vladimir S. Airapetian ${ }^{1}$, Alex Glocer ${ }^{1}$, George V. Khazanov ${ }^{1}$, R. O. P. Loyd ${ }^{2}$, Kevin France ${ }^{2}$, Jan Sojka ${ }^{3}$, William C. Danchi ${ }^{1}$, and \\ Michael W. Liemohn ${ }^{4}$ \\ ${ }^{1}$ NASA/GSFC, Greenbelt, MD, USA \\ ${ }^{2}$ University of Colorado/LASP, Boulder, CO, USA \\ ${ }^{3}$ Utah State University, Logan, UT, USA \\ ${ }^{4}$ University of Michigan, Ann Arbor, MI, USA \\ Received 2016 November 22; revised 2016 December 27; accepted 2016 December 29; published 2017 February 6
}

\begin{abstract}
Atmospheres of exoplanets in the habitable zones around active young G-K-M stars are subject to extreme X-ray and EUV (XUV) fluxes from their host stars that can initiate atmospheric erosion. Atmospheric loss affects exoplanetary habitability in terms of surface water inventory, atmospheric pressure, the efficiency of greenhouse warming, and the dosage of the UV surface irradiation. Thermal escape models suggest that exoplanetary atmospheres around active K-M stars should undergo massive hydrogen escape, while heavier species including oxygen will accumulate forming an oxidizing atmosphere. Here, we show that non-thermal oxygen ion escape could be as important as thermal, hydrodynamic $\mathrm{H}$ escape in removing the constituents of water from exoplanetary atmospheres under supersolar XUV irradiation. Our models suggest that the atmospheres of a significant fraction of Earth-like exoplanets around M dwarfs and active K stars exposed to high XUV fluxes will incur a significant atmospheric loss rate of oxygen and nitrogen, which will make them uninhabitable within a few tens to hundreds of Myr, given a low replenishment rate from volcanism or cometary bombardment. Our non-thermal escape models have important implications for the habitability of the Proxima Centauri's terrestrial planet.
\end{abstract}

Key words: hydrodynamics - planets and satellites: atmospheres - planets and satellites: magnetic fields - radiation mechanisms: general - stars: activity - stars: late-type

\section{Introduction}

Recent detection of terrestrial-type exoplanets with approximately Earth sizes and masses within the climatological habitable zones (CHZs) provides the first glimpse into the potential habitability of these worlds (Kopparapu et al. 2016). The classical definition of the CHZ invokes the total amount of thermal energy emitted by stellar photospheres received by the planet at a given point in time, but largely ignores the impact of the star's non-thermal emission and the level of its magnetic energy on the thickness of an exoplanet's atmosphere and its habitability. Our Sun is a relatively mild magnetic star, but its impact on all levels of the Earth's atmosphere varies dramatically during solar activity cycles (Schrijver et al. 2015). In particular, solar flares perturb the Earth's magnetosphere and ionosphere through electromagnetic radiation, energetic particles, and coronal mass ejections (CMEs). During a flare, the solar flux in X-ray UV (XUV, 20-300 A) band increases by a factor of 1000 and in the extreme UV (EUV, 300-1215 $\AA$ ) band by up to a factor of 20 (Woods \& Rottman 2005). At heights greater than $200 \mathrm{~km}$, such high $\mathrm{XUV}$ and EUV emission (abbreviated here as XUV) and particles precipitating from the magnetosphere ionize gas, producing photo and secondary electrons, an important contributor to outflows of charged atmospheric particles on Earth, Venus, and Mars (Yamauchi \& Wahlund 2007; Lundin 2011; Collinson et al. 2016). During strong flares, electron density in Earth's atmosphere at heights of $60 \mathrm{~km}$ and above varies over one order of magnitude (Kutiev et al. 2013; Mlynczak et al. 2014). Carrington-class solar flares are usually associated with fast and dense CME events that are capable of eroding and compressing the Earth's magnetosphere to a standoff distance as little as 1.25 Earth radii, producing catastrophic effects for our civilization by depositing substantial energy through Joule heating and inducing strong geomagnetic currents (Ngwira et al. 2014; Airapetian et al. 2015, 2016).

Recent reconstructions of XUV fluxes from a number of young $\mathrm{G}, \mathrm{K}, \mathrm{M}$ dwarfs suggest that their coronae and transition regions produce intensive non-thermal emission (Loyd et al. 2016; Youngblood et al. 2016). X-ray luminosities from very young ( $100 \mathrm{Myr}$ ) rapidly rotating K-G stars are up to a factor of over 1000 greater than the current Sun's X-ray luminosity (Tu et al. 2016). As solar-type stars age, their X-ray and EUV luminosities decrease (Güdel et al. 1997). The X-ray luminosities from young and active $\mathrm{M}$ dwarfs are over two orders of magnitude greater than the Sun, but are characterized by compact CHZs because of their lower luminosities (Kopparapu et al. 2016). The sizes of the CHZs for M dwarfs vary between 0.05 and $0.16 \mathrm{au}, 6-20$ times smaller than the $\mathrm{CHZs}$ of $\mathrm{K}$ and $\mathrm{G}$ types stars. Thus, exoplanets around red dwarfs should be exposed to XUV fluxes up to two orders of magnitude larger than those around active solar-type $\mathrm{G}$ and $\mathrm{K}$ stars.

In Figure 1, we present the reconstructed spectral energy distribution (SED) of the current Sun at the average level of activity (between solar minimum and maximum with total flux, $F_{0}(5-1216 \AA)=5.6 \mathrm{erg} \mathrm{cm}^{-2} \mathrm{~s}^{-1}$; yellow dotted line), the X5.5 solar flare occurred on 2012 March 7 (blue line), the young Sun at $0.7 \mathrm{Gyr}$ (yellow solid line), and an inactive M1.5 red dwarf, GJ 832 (red line). The spectra for the current Sun and the solar X5.5 flare in the XUV band $(0.5-10 \AA)$ are constructed from the Solar Dynamic Observatory/EVE instrument data. The Vacuum Ultraviolet (VUV) contribution of the total radiative output is obtained by implementing the Flare Irradiance Spectral Model (FISM; Chamberlin et al. 2008), which represents an empirical model developed for space 


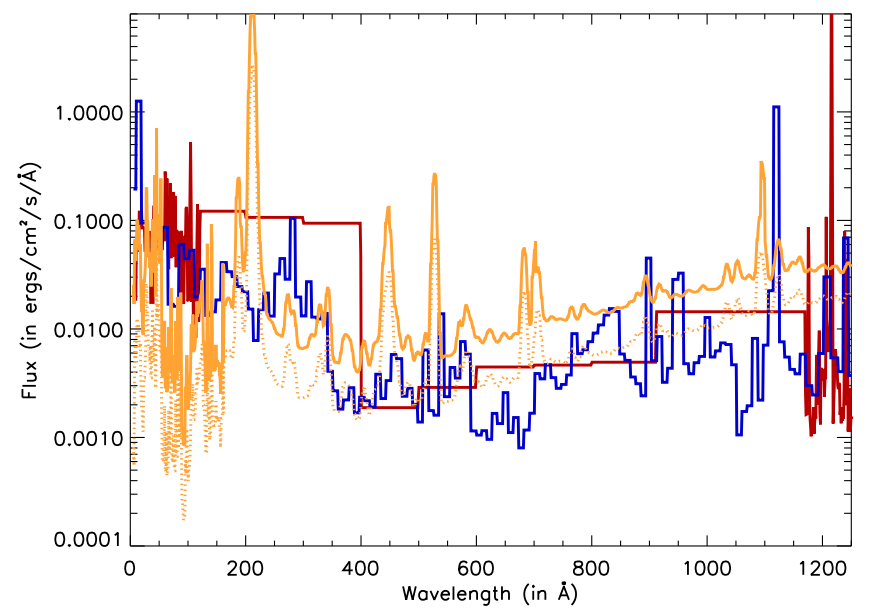

Figure 1. Spectral energy distribution (SED); reconstructed for the solar X5.4 flare (blue curve) and the young Sun's SED (orange curve) and the quiet Sun at the average magnetic activity (dotted orange curve) scaled to 1 au and GJ 832 SED (red curve) scaled to 0.16 au.

weather applications that estimates the solar irradiance at wavelengths from 1 to $1900 \AA$ at $10 \AA$ resolution with a time cadence of $60 \mathrm{~s}$. We also reconstructed the XUV spectrum of a moderately old and inactive M1.5 dwarf, GJ 832, that hosts a super-Earth planet at 0.16 au using the Measurements of the Ultraviolet Spectral Characteristics of Low-mass Exoplanetary Systems (MUSCLES) Treasury Survey data (Loyd et al. 2016). Finally, to approximate the spectrum of the young Sun at $0.7 \mathrm{Gyr}$, we used the data obtained from the parameterization of the two young solar analogs of the Sun at around $0.7 \mathrm{Gyr}, k^{1}$ Cet, and EK Dra (Claire et al. 2012). The total XUV flux from the young Sun and the red dwarf are $8.3 F_{0}$ (at $1 \mathrm{au}$ ) and $7.7 F_{0}$ (at $0.16 \mathrm{au}$ ), respectively. The XUV flux from the young Sun, and GJ 832 are comparable in magnitude and shape at wavelengths shorter (and including) Ly $\alpha$ emission line. This suggests the contribution of X-type flare activity flux is dominant in the "quiescent" fluxes from the young Sun and inactive $\mathrm{M}$ dwarfs. This conclusion is consistent with the estimate of the frequency of occurrence of energetic X-type flares from the young Sun derived from Kepler data (Airapetian et al. 2016). Because X-type white-light flares from the Sun are usually associated with fast and energetic CMEs, it is reasonable to assume that the young Sun and quiet dwarfs should produce frequent CMEs (and associated solar energetic particle events). The dynamic pressure from CMEs can significantly modify the planetary magnetic field and cause energy dissipation in its polar regions (Airapetian et al. 2015).

\section{Effects of XUV-EUV Driven Mass Loss of $\mathbf{O}^{+}$}

XUV radiation induces non-thermal heating via photoabsorption and photoionization raising the temperature of the exosphere, and therefore its pressure scale height. At high XUV fluxes, this process initiates hydrodynamic atmospheric escape of neutral atmospheric species, with the loss rate dependent on the molecular mass of atmospheric species. Hydrogen, as the lightest component, escapes more readily than any other species by this mechanism (Lammer et al. 2008; Tian et al. 2008). For the environments of active solar-type stars and $M$ dwarfs, much of the hydrogen likely escapes from a planet's atmosphere during the system's early evolution, leaving behind an atmosphere enriched in heavier elements such as $\mathrm{N}$ and $\mathrm{O}$.
These species are difficult to remove unless dense and fast stellar winds or the processes of photochemical escape are invoked (Lammer et al. 2003; Lee et al. 2015; Lichtenegger et al. 2016). Therefore, models of atmospheric ionization and loss via non-thermal mechanisms are critical for predicting the evolution of oxygen and nitrogen-rich atmospheres as well the efficiency of atmospheric loss of water as a critical factor of exoplanetary habitability.

In the region above an Earth-size planet's exobase, the layer where collisions are negligible, the incident XUV flux ionizes atmospheric atoms and molecules and produces photoelectrons. The upward propagating photoelectrons outrun ions in the absence of a radially directed polarization electric field and forms the charge separation between electrons and atmospheric ions. Thus, a radially directed polarization electric field is established that enforces the quasi-neutrality and zero radial current. For ionospheric ions with energies over $10 \mathrm{eV}$, the polarization electric field cancels a substantial part of the Earth's gravitational potential barrier, greatly enhancing the flux of escaping ions and forming an ionospheric outflow.

Modeling of these effects requires coupling the hydrodynamic ion and superthermal electron kinetic equations with the polarization electric field (Liemohn et al. 1997). We apply this approach to couple the ion hydrodynamics of the Polar Wind Outflow Model (PWOM) to the latest version of the SuperThermal Electron Transport (STET) code (Glocer et al. 2009, 2012; Khazanov 2011; Khazanov et al. 2015). Full details of the model coupling will appear in a separate publication (Glocer et al. 2016). To treat the XUV driven photoelectron production and transport properly, we apply STET to calculate the superthermal particle population formed via photoionization and its collisional coupling with the thermal population and the neutral atmosphere.

Our coupled PWOM and STET model uses MSIS-90 (mass spectrometer and incoherent scatter) empirical model developed for the Earth atmosphere (Hedin 1987) as an input for PWOM and STET to obtain the neutral densities including O, $\mathrm{O}_{2}$, and $\mathrm{N}_{2}$ and temperatures. In this study, we did not consider the processes of photolysis of water molecules that can provide atomic oxygen through formation of hydroxyl molecules and hydrogen atoms that thermally escape from the atmosphere. In essence, we assume that water in the lower atmosphere is photolyzed into $\mathrm{H}, \mathrm{O}$, and $\mathrm{O}_{2}$ before reaching the upper atmospheric domain of our simulations.

To properly treat photodissociation and photoionization of major species we used the XUV emission input in the range between 5 and $1750 \AA$. Specifically, $\mathrm{O}^{+}$ions form due to photoionization of atomic oxygen via photons with wavelengths $~ 300-600 \AA$ and collisions with photoelectrons. The processes of collisional ionization of $\mathrm{O}$ and $\mathrm{N}$ atoms due to precipitating electrons formed during magnetic storms from a host star provide additional sources of $\mathrm{O}^{+}$production and will be modeled in the near future.

We have developed four models with the stellar XUV input flux expressed in terms of the total XUV flux, $F_{0}$, of the Sun at the average level of magnetic cycle. Figure 2 shows the steadystate solution for the production of superthermal photoelectrons with the energies extending to $70 \mathrm{eV}$. A prominent emission feature of the XUV spectra in Figure 1, the He II $304 \AA$ resonance emission line, produces a series of spikes of photoelectrons within the $20-30 \mathrm{eV}$ energy range due to the various ionization states of atmospheric constituents. The figure 


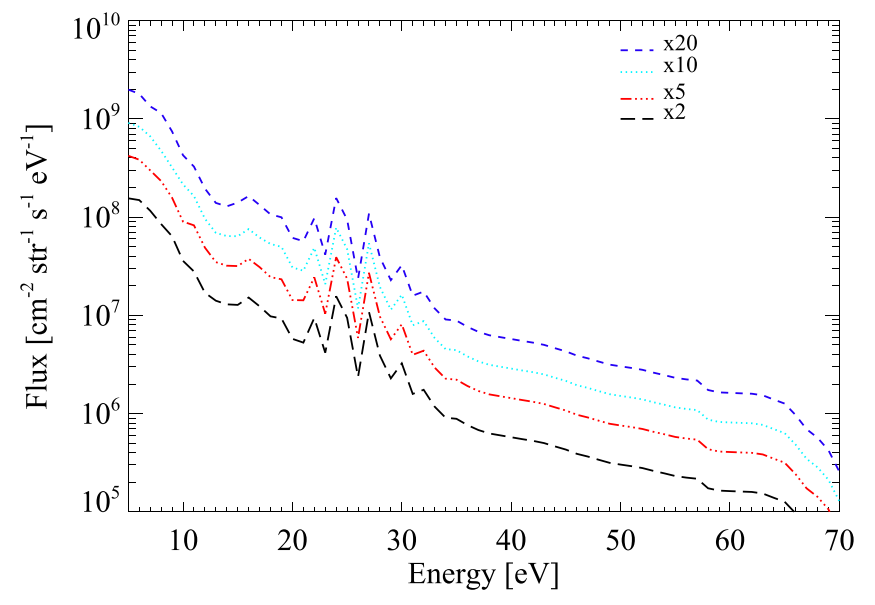

Figure 2. Flux of the primary photoelectrons produced due to XUV/EUV emission at $\mathrm{x} 2, \mathrm{x} 5, \mathrm{x} 10$, and $\mathrm{x} 20 F_{\mathrm{o}}$ as a function of electron energy.

suggests that the photoelectron flux increases approximately linearly with the input XUV flux. PWOM then uses STET's representation of the superthermal electrons to model the ionized atmosphere escape rates. Our model calculates the mass outflow rate of $\mathrm{H}^{+}$and $\mathrm{O}^{+}$ions in the exo-Earth's atmosphere along an open single magnetic field line of the polar region at heights between 200 and $6000 \mathrm{~km}$.

To estimate the extent of the polar region, we use the results of our early Earth's global magnetospheric MHD simulations that can serve as the proxy of an Earth analog, an Earth-sized planet. The XUV flare flux at $10 F_{0}$ corresponds to the associated Carrington-type CME event with the high dynamic pressure and large interplanetary magnetic field (IMF). Assuming that the IMF field is opposite to the dayside magnetospheric field orientation, this event will open $\sim 70 \%$ of the planetary magnetic field (Airapetian et al. 2016). We then calculated the steady-state outflow rate of $\mathrm{O}^{+}$ions driven by the input XUV flux and the value of the neutral temperature specified at the exospheric base at $200 \mathrm{~km}$. In the current model, the neutral temperature at the atmospheric base is fixed. However, due to the absorption of the enhanced XUV flux, the neutral temperature should increase. For example, the absorption of the XUV flux in the range of inputs between (5-20) $F_{0}$ causes the base temperature (Smithtro \& Sojka 2005; Tian et al. 2008 ) at $200 \mathrm{~km}$ to rise from $1000 \mathrm{~K}$ at $1 F_{0}$ to 2000 at $5 F_{0}$ and reaches $\sim 3000 \mathrm{~K}$ at $20 F_{0}$. Currently, our WPOM + STET model is not coupled to the ionosphere-thermosphere model and the effects of enhanced base neutral temperature on outflow rate cannot be treated self-consistently because of the computational expense.

In order to evaluate the effect of the base temperature on the $\mathrm{O}^{+}$outflow rate, we calculated two escape models for the XUV flux of $10 F_{0}$ for these two exobase temperatures. We find that as we increase the base temperature by a factor of 2 , the resulting $\mathrm{O}^{+}$outflow rates increase by a factor of 10 .

We then calculated the four models for an exoplanet with the same atmospheric properties and mass as the current Earth (Earth twin) irradiated by XUV fluxes at 2, 5, 10, and $20 F_{0}$, respectively at $T_{\text {base }}=2000 \mathrm{~K}$. Because the base temperature of the neutral atmosphere is $>2000 \mathrm{~K}$ at fluxes higher than $10 F_{0}$, the model output represents a lower limit on the outflow rates along the magnetic field line. The total loss rate of $\mathrm{O}^{+}$at $h=1000 \mathrm{~km}$ is found from the integration of this value over the whole area, $S$, region of the polar region as $\dot{M}=\rho V S$,

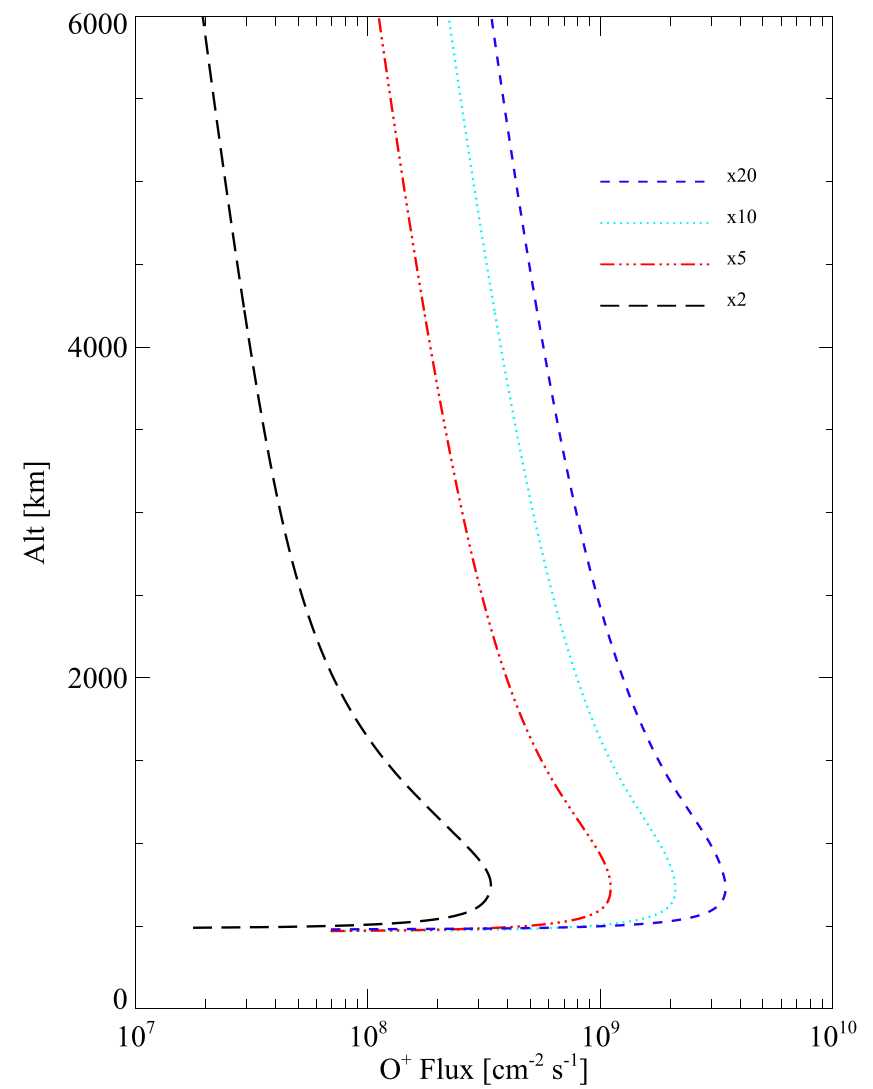

Figure 3. Mass-loss rate of oxygen ions from the Earth atmosphere due to XUV and EUV irradiation from the young Sun at $F=2$ (long dash), 5 (dashdot), 10 (dot), 20 (short dash).

where $\rho$ is the density of the oxygen ions at that altitude, $V$ is the $\mathrm{O}^{+}$ion bulk velocity. Figure 3 also shows that the mass loss of oxygen ions increases roughly linearly with the solar flux and reaches $\sim 400 \mathrm{~kg} \mathrm{~s}^{-1}$ for $F=20 F_{\text {Sun }}$. This estimate does not account for a number of effects typically contributing to the ion escape during space weather events associated with large solar flares. At XUV fluxes $\sim 20 F_{0}$, the base temperature rises to $3000 \mathrm{~K}$. Thus, the expected mass-loss rate will increase by a factor of $\sim 4$ with respect to the model output at the base temperature of $2000 \mathrm{~K}$ with the total $\mathrm{O}^{+}$loss rate of $\sim 1600 \mathrm{~kg} \mathrm{~s}^{-1}$. This mass-loss rate will be further increased by precipitated energetic electrons from the day and night sides of the Earth's magnetosphere. This input efficiently produces secondary superthermal electrons due to collisional ionization of species in the ambient ionosphere (Strangeway et al. 2005). In nitrogen-rich exoplanetary atmospheres, XUV fluxes along with photo and secondary electrons ionize atomic nitrogen along with oxygen. The observations of ionospheric outflows driven by geomagnetic storms show that the outflow rate of nitrogen ions may be comparable to the loss rate of $\mathrm{O}^{+}$, although caution should be exercised when using these observational results for our analysis because the mass peaks of $\mathrm{O}^{+}$and $\mathrm{N}^{+}$are not well resolved (Yau et al. 2007). Future missions, including the recently proposed ESCAPE mission, should resolve the question of nitrogen loss rate as a function of various environmental factors. Thus, the loss rate is scaled with the input flux as

$$
\dot{M}\left(\text { in } \mathrm{g} \mathrm{s}^{-1}\right) \sim 1.6 \times 10^{4} F_{\mathrm{XUV}}\left(\text { in erg } \mathrm{cm}^{-2} \mathrm{~s}^{-1}\right) .
$$

This loss rate is comparable to the thermal loss of hydrogen at XUV fluxes $\sim 20 F_{0}$ at the inner edge of $\mathrm{CHZ}$ if radiative 
cooling and the transition from hydrodynamic to Jeans escape are accounted for an $\sim 1$ Earth-mass planet (Owen \& Mohanty 2016). The non-thermal loss via $\mathrm{H}^{+}$escape driven by the polarization electric field mediated by photoelectrons can also be potentially important in removing hydrogen and helium in the primary atmospheres of Earth analogs. Oxygen ion loss at the rate prescribed by Equation (1) will remove Earth's oxygen at the surface pressure of 1 bar, $\sim 1.2 \times 10^{18} \mathrm{~kg}$ atmosphere within 24.2 Myr if it is not replenished by $\mathrm{CO}_{2}$ through tectonic and volcanic outgassing.

Application of Equation (1) to the super-Earth around an inactive red dwarf, GJ 832 (Bailey et al. 2009), exposed to the XUV flux of $10 F_{0}$ at 0.16 au suggests that the planet with 1 bar Earth-like atmospheric composition could lose oxygen through $\mathrm{O}^{+}$escape within $50 \mathrm{Myr}$. Because $\mathrm{M}$ dwarfs remain magnetically active over Gyr timescales, their transition regions and coronae are expected to produce high levels $\left(>10 F_{0}\right)$ of XUV fluxes at the inner edges of CHZs. Thus, for low luminosity mid- and late-M dwarfs possessing compact $\mathrm{CHZs}$ $(<0.1 \mathrm{au})$, desiccation and overall atmospheric loss present challenging conditions for planetary habitability at the timescales from a few to a few hundred Myr. Our result implies that the $\mathrm{CHZ}$ definition can only be applied to mid-age K-G mainsequence stars for which the XUV fluxes at the distances of their CHZs are small enough $\left(<5 F_{0}\right)$ to be significant for the atmospheric loss at evolutionary timescales. For higher fluxes, atmospheric loss rates are high enough to affect atmospheric evolution, and to reflect this impact, it may be useful to expand the definition of CHZs to Space Weather Affected Habitable Zones (SWAHZs).

\section{Conclusions}

Our model provides important constraints on the mass loss of oxygen driven by XUV fluxes from Proxima Cen from the recently discovered Earth-like planet, Proxima Cen $b$ (Anglada-Escudé et al. 2016). The host star, Proxima Cen is characterized by an X-ray flux comparable to the Sun at the maximum of solar activity and at the location of Proxima $b$, $0.05 \mathrm{au}$, the flux should be enhanced by a factor of $60 F_{0}$ (Ribas et al. 2016). Tidal effects are significant for close-in planetary evolution (Driscoll \& Barnes 2015). The uncertainty in the orbital inclination angle to the line of sight, $M \sin i$, implies that the true planet's mass can be either comparable to Earth's (Earth-like) or be a few times larger (1-5 times; super-Earth). For an Earth-like planet, Proxima b's slow rotation rate should not have a significant impact on magnetic field generation, as this is mostly influenced by the internal heat flux that controls the mantle convection rather than a dynamo (Christensen \& Aubert 2006; Stamenković et al. 2012). Thus, we can expect that the planet has an Earth-like dipole magnetic field. However, if the planet is super-Earth size, full mantle convection becomes less efficient, so that heat in deep interiors is transferred by conduction rather than convection. This reduces the efficiency of volcanic activity and the magnetic dynamo in massive terrestrial-type planets including the superEarth around GJ 832 discussed in Section 2 (Stamenković et al. 2012).

If we apply the derived scaling of the loss rate with $F_{\mathrm{XUV}}$, then the escape time of a 1 bar oxygen-rich atmosphere on Proxima Cen $b$ is expected to be $\sim 10$ Myr. Thus, radiative forcing should play an important role in oxygen ion loss via enhanced ionization and heating of the lower atmosphere, and in the formation of ambipolar electric fields in the upper atmosphere. Steep electron temperature gradients in the upper atmosphere should produce strong ambipolar fields above the exobase, and initiate increased outflow of $\mathrm{O}^{+}$and $\mathrm{N}^{+}$ions. Also, a recent MHD model of the stellar environment of Proxima Centauri suggests that the close-in Earth-size planet with an Earth-like magnetic moment should be subject to wind pressure of $\sim 1000$ times greater than that exerted at Earth, compressing the planetary magnetic field, and thus reducing magnetospheric distance by a factor of 3 (Garraffo et al. 2016). Ionospheric electric currents induced by the compressed planetary magnetic field efficiently dissipate energy at the rate of $\sim 10^{4} \mathrm{erg} \mathrm{cm}^{-2} \mathrm{~s}^{-1}$ through Joule resistive heating in the polar cap region. Observations of Earth's response to solar wind pressure show that Joule heating at the rate of $10 \mathrm{erg} \mathrm{cm}^{-2} \mathrm{~s}^{-1}$ (Cai et al. 2014) significantly modifies the electrodynamics and thermodynamics of the coupled magnetosphere-ionosphere-thermosphere system by increasing neutral and ion temperatures and densities ( $\mathrm{Lu}$ et al. 2010; Anderson et al. 2013). Additionally, Moore et al. (1997) showed that a solar wind pressure pulse caused significant additional ionospheric outflow at Earth, and this effect has been further quantified by observational statistical studies (e.g., Cully et al. 2003; Lennartsson et al. 2004) and numerical simulation studies (Yu \& Ridley 2009; Damiano et al. 2010; Welling \& Liemohn 2014).

Increased temperature enhances the pressure scale height, and thus provides an additional source of ionospheric heating (besides collisional heating due to radiation and precipitating particles) that will increase the escape rate of oxygen, nitrogen, and heavy ions by a factor of 3-5 with respect to the quiet magnetosphere (Strangeway et al. 2005; Yau et al. 2007). In addition, charge exchange due to a dense and fast stellar wind will provide an efficient process for additional loss of hydrogen, oxygen and nitrogen from the planetary atmosphere (Lammer et al. 2008; Lichtenegger et al. 2016). In the case of the super-Earth scenario, the planet should have a tenuous atmosphere due to the lack of volcanic outgassing and no appreciable dipole magnetic field as discussed earlier. Strong crustal magnetic field sources, as found on Mars (Acuña et al. 1999), could shield the upper atmosphere from direct solar wind scavenging, but the effect is only in the range of perhaps a 50\% reduction (e.g., Fang et al. 2010; Ma et al. 2014; Brecht et al. 2016). Further, the effects of charge exchange should be significant to its atmospheric loss. Thus, Proxima Cen $b$ appears to have a low probability of being habitable in either scenario.

We conclude that atmospheres of Earth-sized planets within respective SWAHZs should be vulnerable to the high XUV fluxes, making close-in planets around low luminosity $M$ dwarfs uninhabitable within a few to hundred Myr, assuming minimal replenishment from volcanic outgassing and bombardment by comets. However, it is expected that delivery of water via comets was efficient in the first $0.5 \mathrm{Gyr}$ during the Late Heavy Bombardment, and thus should be important for young planetary systems. Our scaling law of the mass-loss rate of $\mathrm{O}^{+}$ with the incident XUV flux suggests that terrestrial-type planets may be habitable if they are located at $\geqslant 0.3$ au or at the outer edges of $\mathrm{CHZ}$, and therefore a very efficient greenhouse warming should be available to support liquid surface water. Kepler data suggest that the occurrence rates of terrestrial planets (with radii between 1 and $4 R_{\text {Earh }}$ ) are $0.3,0.2$, and 0.1 
planet per star for M, K, and G dwarfs, respectively (Mulders et al. 2015). The inner edge of the $\mathrm{CHZ}$ for $\mathrm{K}$ dwarfs are about 3 times further away from their hosts stars as compared to $\mathrm{M}$ dwarfs and the corresponding XUV fluxes are one order of magnitude smaller for an equivalent XUV luminosity. This implies that the best candidates for truly habitable Earth-like planets will be those in CHZs around middle-aged (over $1 \mathrm{Gyr}$ ) mid-K to $G$ dwarfs that provide mild space weather environments.

Future observations with The James Web Space Telescope $(J W S T)^{5}$, large ground-based telescopes, and future space telescopes such as The Habitable Exoplanet Imaging Mission $(H a b E x)^{6}$ and the Large UltraViolet Optical InfraRed (LUVOIR) ${ }^{7}$ Surveyor may provide conclusive answers on the habitability of these exotic worlds.

This paper is dedicated to the memory of Dr. Piers Sellers, who served as a great inspiration for our ongoing interdisciplinary team project "Mission to Young Earth 2.0". This study was supported by the internal Science Task Group funds administered by NASA Goddard Space Flight Center's Sciences and Exploration Directorate. The authors also wish to thank the anonymous referee for the constructive comments and suggestions.

\section{References}

Acuña, M. H., Connerney, J. E. P., Ness, N. F., et al. 1999, Sci, 284, 790

Airapetian, V., Glocer, A., \& Danchi, W. 2015, in Proc. 18th Cambridge Workshop on Cool Stars, Stellar Systems, and the Sun, ed. G. van Belle \& H. Harris (arXiv:1409.3833)

Airapetian, V., Glocer, A., Gronoff, G., Hébrard, E., \& Danchi, W. 2016, NatGe, 9, 452

Anderson, C., Kosch, M. J., Nicolls, M. J., \& Conde, M. 2013, JASTP, 105, 313 Anglada-Escudé, G., Amado, P. J., Barnes, J., et al. 2016, Natur, 536, 437 Bailey, J., Butler, R. P., Tinney, C. G., et al. 2009, ApJ, 690, 743 Brecht, S. H., Ledvina, S. A., \& Bougher, S. W. 2016, JGRA, 121, 10190 Cai, L., Aikio, A. T., \& Nygrén, T. 2014, JGR, 119, 10440

Chamberlin, P. C., Woods, T. N., \& Eparvier, F. G. 2008, SpWea, 6, S05001 Christensen, U. R., \& Aubert, J. 2006, GeoJ, 166, 97

Claire, M. W., Sheets, J., Cohen, M., et al. 2012, ApJ, 757, 95

Collinson, G. A., Frahm, R. A., Glocer, A., et al. 2016, GeoRL, 43, 5926

Cully, C. M., Donovan, E., Yau, A. W., \& Arkos, G. G. 2003, JGR, 108, 1093

Damiano, P. A., Brambles, O. J., Lotko, W., et al. 2010, JGR, 115, A00J07
Driscoll, P. E., \& Barnes, R. 2015, AsBio, 15, 739

Fang, X., Liemohn, M. W., Nagy, A. F., Luhmann, J. G., \& Ma, Y. 2010, Icar, 206, 130

Garraffo, C., Drake, J. J., \& Cohen, O. 2016, ApJL, 833, L4

Glocer, A., Khazanov, G. V., \& Liemohn, M. 2016, JGR, submitted

Glocer, A., Kitamura, N., Tóth, G., \& Gombosi, T. 2012, JGR, 117, A04318

Glocer, A., Toth, G., Gombosi, T., \& Welling, D. 2009, JGR, 114, A12203

Güdel, M., Guinan, E. F., \& Skinner, S. L. 1997, ApJ, 483, 947

Hedin, A. E. 1987, JGR, 92, 4649

Khazanov, G. 2011, Kinetic Theory of the Inner Magnetospheric Plasma, Vol. 372 (Berlin: Springer)

Khazanov, G. V., Tripathi, A. K., Sibeck, D., et al. 2015, JGR, 120, 9891

Kopparapu, R. K., Wolf, E. T., Haqq-Misra, J., et al. 2016, ApJ, 819, 84

Kutiev, I., Tsagouri, I., Perrone, L., et al. 2013, JSWSC, 3, A06

Lammer, H., Kasting, J. F., Chassefiere, E., et al. 2008, SSRv, 139, 399

Lammer, H., Lichtenegger, H. I. M., Kolb, C., et al. 2003, Icar, 165, 9

Lee, Y., Combi, M. R., Tenishev, V., et al. 2015, GeoRL, 42, 9015

Lennartsson, O. W., Collin, H. L., \& Peterson, W. K. 2004, JGR, 109, A12212

Lichtenegger, H. I. M., Kislyakova, K. G., Odert, P., et al. 2016, JGR, 121,4718

Liemohn, M. W., Khazanov, G. V., Moore, T. E., \& Guiter, S. M. 1997, JGR, 102,7523

Loyd, R. O. P., France, K., Youngblood, A., et al. 2016, ApJ, 824, 102

Lu, G., Mlynczak, M. G., Hunt, L. A., Woods, T. N., \& Roble, R. G. 2010, JGR, 115, A05306

Lundin, R. 2011, Space Science Ser. of ISSI, 37, 309

Ma, Y., Fang, X., Russell, C. T., et al. 2014, GeoRL, 41, 6563

Mlynczak, M. G., Hunt, L. A., Mertens, C. J., et al. 2014, GeoRL, 41, 2508

Moore, T. E., Chappell, C. R., Chandler, M. O., et al. 1997, Sci, 277, 349

Mulders, G., Pascucci, I., \& Apai, D. 2015, ApJ, 798, 112

Ngwira, C. M., Pulkkinen, A., Kuznetsova, M. M., \& Glocer, A. 2014, JGR, 119,4456

Owen, J. E., \& Mohanty, S. 2016, MNRAS, 459, 4088

Ribas, I., Bolmont, E., Selsis, F., et al. 2016, A\&A, 596, A111

Schrijver, C. J., Kauristie, K., Alan, D., et al. 2015, AdSpR, 55, 2745

Smithtro, C. J., \& Sojka, J. J. 2005, JGR, 110, A08306

Stamenković, V., Noak, L., Breuer, D., \& Spohn, T. 2012, ApJ, 748, 22

Strangeway, R. J., Ergun, R. E., Su, Y.-J., Carlson, C. W., \& Elphic, R. C. 2005, JGR, 110, A03221

Tian, F., Kasting, J. F., Liu, H.-L., \& Roble, R. G. 2008, JGR, 113, E05008

Tu, L., Johnstone, C., Gudel, M., \& Lammer, H. 2016, A\&A, 577, L3

Welling, D. T., \& Liemohn, M. W. 2014, JGRA, 119, 2691

Woods, T. N., \& Rottman, G. 2005, SoPh, 230, 375

Yamauchi, M., \& Wahlund, J.-E. 2007, AsBio, 7, 783

Yau, A. W., Abe, T., \& Peterson, W. K. 2007, JASTP, 69, 1936

Youngblood, A., France, K., Parke Loyd, R. O., et al. 2016, ApJ, 824, 101

Yu, Y., \& Ridley, A. J. 2009, AnGeo, 27, 4391

\footnotetext{
5 http://www.jwst.nasa.gov/

6 http://www.jpl.nasa.gov/habex/

7 http://asd.gsfc.nasa.gov/luvoir/
} 